\title{
Erratum: Guaranteed Convergence of a Regularized Kohn-Sham Iteration in Finite Dimensions [Phys. Rev. Lett. 123, 037401 (2019)]
}

\author{
Markus Penz, Andre Laestadius, Erik I. Tellgren, Michael Ruggenthaler, and Paul E. Lammert
}

(Received 1 October 2020; published 9 December 2020)

DOI: 10.1103/PhysRevLett.125.249902

The central argument for convergence of the "MYKSODA iteration scheme" introduced in our article [1] contains a critical mistake that we hereby want to correct. The claim was that, because of $(1 / 2 \varepsilon)\left\|x_{i+1}-x_{i}\right\|^{2} \leq e_{i}-e_{i+1} \rightarrow 0$ in Eq. (19), the sequence of quasidensities $\left(x_{i}\right)_{i}$ also converges. This claim is wrong since it does not show the necessary Cauchy property $\left\|x_{m}-x_{n}\right\| \rightarrow 0$ for all $m, n \rightarrow \infty$. Convergence could be secured, on the other hand, in cases where $e_{i}-e_{i+1} \leq r^{i}$ with $0<r<1$ because then $\left\|x_{i+1}-x_{i}\right\| \leq C \sqrt{r}^{i}$ and the summability of the geometric series would give the Cauchy property for $\left(x_{i}\right)_{i}$. Yet, such a condition for the convergent energy sequence $\left(e_{i}\right)_{i}$ is in general not available. The corrigendum given below proceeds along a different path that effectively takes degeneracies of the ground state into account. First, it is shown that the sequence of quasidensities $\left(x_{i}\right)_{i}$ is bounded. Since we operate in a finite-dimensional setting, this means by the Bolzano-Weierstrass theorem that the sequence has at least one convergent subsequence. We then show that all such subsequences converge to ground-state quasidensities of the regularized, fully interacting problem. Thereby, the accumulation points of the sequence give the desired solutions, of which there can be more than one in case of degeneracy. Although in this way the algorithm can accommodate multiple valid solutions, we cannot be sure that we map out all possible solutions. The selection of specific solutions might still depend on the initial value $x_{0}$.

While the MYKSODA iteration scheme remains precisely the same, the statement following it has to be modified. We prove that, for any convergent subsequence $x_{\alpha(i)} \rightarrow z$, the corresponding potential sequence $v_{\alpha(i)}$ converges to the correct Kohn-Sham (KS) potential that reproduces $z$ in the regularized reference problem. We will now repeat the last part of the proof that needs to be adjusted.

Corrected convergence proof.-Up to Eq. (19), all statements stay intact-just the inference of convergence right after Eq. (19) is erroneous. The $F_{\varepsilon}$ increases like $\|x\|^{2}$ asymptotically by construction [cf. the regularization procedure in Eq. (5)] because $F(x)=+\infty$ whenever $x$ is outside the bounded $\tilde{X}$. Since $e_{i}=F_{\varepsilon}\left(x_{i}\right)+\left\langle v, x_{i}\right\rangle \leq e_{1}$ for all $i$, this means that $\left(x_{i}\right)_{i}$ must already be bounded. This translates to boundedness of $\left(v_{i}\right)_{i}$ defined by step (a) because $\nabla F_{\varepsilon}, \nabla F_{\varepsilon}^{0}$ are Lipschitzbounded (with constant $\varepsilon^{-1}$; Barbu and Precupanu [2][Corollary 2.59]). We can then argue that $\left\|\nabla F_{\varepsilon}\left(x_{i}\right)+v\right\| \rightarrow 0$ and $\left\|\nabla F_{\varepsilon}^{0}\left(x_{i}\right)+v_{i+1}\right\| \rightarrow 0$, exactly like in the original proof. This means $v=-\lim _{i} \nabla F_{\varepsilon}\left(x_{i}\right)$ and for any accumulation point $z$ of $\left(x_{i}\right)_{i}$ (Bolzano-Weierstrass guarantees that there is at least one) there must be a convergent subsequence $x_{\alpha(i)} \rightarrow z$ such that $v=-\lim _{i} \nabla F_{\varepsilon}\left[x_{\alpha(i)}\right]=-\nabla F_{\varepsilon}(z)$. By inversion we get $z \in \bar{\partial} E_{\varepsilon}(v)$, which means that $z$ is the correct ground-state quasidensity of the regularized full problem. Finally, $\lim _{i} v_{\alpha(i+1)}=-\lim _{i} \nabla F_{\varepsilon}^{0}\left[x_{\alpha(i)}\right]=-\nabla F_{\varepsilon}^{0}(z)=v_{\mathrm{KS}}^{\varepsilon}$ is the KS potential that exactly reproduces this $z$ in the regularized reference problem. A different accumulation point with its associated convergent subsequence $\left[x_{\beta(i)}\right]_{i}$ will lead to another possible ground-state quasidensity of the regularized full problem in the case of degeneracy. Then the procedure assigns it the appropriate KS potential as the limit of $\left[v_{\beta(i)}\right]_{i}$ that reproduces the same quasidensity in the regularized reference problem. In this manner, the whole sequence $\left(x_{i}\right)_{i}$ partitions into convergent subsequences that have ground-state quasidensities as limit points.

Note that it is still true that the distance between successive terms goes to zero, i.e., $\left\|x_{i+1}-x_{i}\right\| \rightarrow 0$. This means that no isolated accumulation points are possible. But since the ground-state solutions in terms of density matrices $\Gamma$ form a convex set and the mapping $\Gamma \mapsto \rho$ is linear, the set of ground-state (quasi)densities will also be convex and thus corresponds to a whole "accumulation region." The sequence $\left(x_{i}\right)_{i}$ will thus converge to this set of solutions. If the ground-state density is nondegenerate (unique), then $\bar{\partial} E_{\varepsilon}(v)$ is single valued and the whole sequence converges to this point only. It has to be noted that it is not possible to straightforwardly generalize the new, corrected proof to a setting of infinite-dimensional Banach spaces. The question of convergence in such a case is still open.

Another small mistake should be noted: after Eq. (13), two times in Eq. (15), and after Eq. (16), there must be a minus sign in front of $\nabla F_{\varepsilon}^{0}$. The error does not influence any subsequent results. We would also like to point out a misleading feature of Fig. 1, where the impression is given that the minima of the one-dimensional sections through $F+v$ and $F_{\varepsilon}+v$ coincide, which holds true in the special case of the absolute minima but not along an arbitrary direction. 
A. L. and E. I. T. acknowledge support from the Norwegian Research Council through Grants No. 287906 and No. 262695 (CoE Hylleraas Center for Quantum Molecular Sciences). M. R. acknowledges support from the Cluster of Excellence "CUI: Advanced Imaging of Matter" of the Deutsche Forschungsgemeinschaft (DFG), EXC 2056; Project ID 390715994.

[1] M. Penz, A. Laestadius, E. I. Tellgren, and M. Ruggenthaler, Phys. Rev. Lett. 123, 037401 (2019).

[2] V. Barbu and T. Precupanu, Convexity and Optimization in Banach Spaces, 4th ed. (Springer, New York, 2012). 